\title{
Improvement of calcium solubility and bioavailability of calcium-fortified soymilk containing Lactobacillus acidophilus, $L$. casei and L. plantarum.
}

\author{
Anne Lise Tang', Karen Z. Walker ${ }^{1}$, Gisela Wilcox ${ }^{1,3}$, Nagendra P. Shah ${ }^{1}$, Lily \\ Stojanovska ${ }^{1}$
}

1. School of Biomedical Sciences and School of Molecular Sciences, Victoria University, Werribee Campus, PO Box 14428 Melbourne, Victoria 8001, Australia

2. Nutrition and Dietetics Unit, Department of Medicine, Monash University, 246, Clayton

Road, Clayton, Vic 3168, Australia

3. Department of Medicine, Clinical Nutrition \& Metabolism Unit and Body Composition Laboratory, Monash Medical Centre, 246, Clayton Road, Clayton, Vic 3168, Australia

A high intake of dietary calcium through out life helps reduce the risk of osteoporosis. The amount of calcium available to the body depends not only on how much is consumed, but on the body's ability to absorb calcium and retain it. As soymilk contains significantly less calcium than cow's milk; calcium is often added as a fortificant, but it is unclear whether this added calcium is well absorbed. Soybeans, like other legumes contain myo-inositol hexaphosphate (IP6), also known as phytate, that can chelate calcium inhibiting its absorption. Fermenting soy milk with certain lactic acid bacteria can reduce the amount of IP6. These microorganisms produce the enzyme, phytase which hydrolyses IP6 to its lower IPs including; myo-inositol pentaphosphate (IP5), IP4, IP3 and IP1. Furthermore, fermenting soy milk with lactic acid bacteria increases the conversion of isoflavones to the biologically active aglycone form. These biologically active soy isoflavones have important actions in decreasing bone loss.

Our study objective was to enhance calcium bioavailability from calcium-fortified soymilk by fermenting it with 6 strains of Lactobacillus namely L. acidophilus ATCC 4962, ATCC 33200, ATCC 4356, ATCC 4461, L. casei ASCC 290, and L. plantarum ASCC 276 to measure calcium solubility, IP6 and isoflavone content.

Calcium-fortified soymilk, made from soy protein isolate, was inoculated with these bacterial strains, incubated for $24 \mathrm{~h}$ at $37^{\circ} \mathrm{C}$ then stored for $14 \mathrm{~d}$ at $4^{\circ} \mathrm{C}$. Total and soluble calcium were measured using atomic absorption spectrophotometry (AA). IP6 and isoflavone profile were measured using HPLC.

Viability of the strains in the fermented calcium-fortified soymilk was $>7.5 \log _{10} \mathrm{CFU} / \mathrm{g}$ at the end of 24 hours of incubation and this was maintained for $14 \mathrm{~d}$ storage at $4^{\circ} \mathrm{C}$. After $24 \mathrm{~h}$ fermentation, there was a significant increase $(\mathrm{P}<0.05)$ in soluble calcium possibly due to a $\mathrm{pH}$ decrease in the fermented calcium-fortified soymilk. L. acidophilus ATCC 4962 and $L$. plantarum ASCC 276 demonstrated $76.6 \%$ and $70.3 \%$ increase in calcium solubility respectively. The productions of the enzyme phytase during fermentation led to the degradation of IP6 to its lower IPs. Fermentation of calcium-fortified soymilk also increased $(\mathrm{P}<0.05)$ the level of conversion of isoflavones into biologically active aglycones by $\beta-$ glucosidase activity.

Our results show that fermenting calcium-fortified soymilk with L. acidophilus, L. casei and L. plantarum can potentially enhance the bioavailability of calcium from calcium-fortified 
soymilk due to increased calcium solubility, IP6 degradation and isoflavone aglycone enrichment. 